\title{
American Cutaneous Leishmaniasis
}

National Cancer Institute

\section{Source}

National Cancer Institute. American Cutaneous Leishmaniasis. NCI Thesaurus. Code C34769.

The most common form of leishmaniasis that is transmitted through the bite of female phlebotomine sand flies or after exposure to leishmania parasites. It is characterized by skin lesions at the site of insect bite which typically develop within weeks or months after exposure. The lesions typically progress from small papules to open sores with raised borders and central ulcers which can be covered with scales or crust. 